\title{
Um Chatterbot Educacional Baseado em EmotionML
}

\author{
Ramayana A. M. Junior, José F. M. Netto \\ Instituto de Computação - Universidade Federal do Amazonas (UFAM) \\ Manaus - AM - Brasil \\ ramayana@icomp.ufam.edu.br, jnettodicomp.ufam.edu.br
}

\begin{abstract}
This short paper describes a proposal for XML language that unites elements of AIML and EmotionML aiming to develop an educational chatterbot with emotional characteristics.

Resumo. Este short paper é descrita uma proposta de linguagem XML que une elementos de AIML e EmotionML com intuito de desenvolver um chatterbot educacional com características emocionais.
\end{abstract}

\section{Introdução}

Atualmente o grande desafio dos sistemas computacionais é a necessidade de melhorar a interação com os usuários. Um caminho para atingir essa melhora é dotar computadores de aspectos afetivos. Nichele e Jaques pontuam sobre esse assunto: "Atualmente, as interfaces de sistemas computacionais têm sido dotadas de habilidades afetivas, a fim de interagir de maneira mais natural e antropomórfica com o usuário." [Nichele e Jaques 2013].

Uma abordagem que vem sendo utilizada para concretizar este objetivo é a combinação de Agentes inteligentes com Chatterbots. Segundo Sganderla et al. (2003) os chatterbots servem-se da ideia básica da interação com as pessoas para dar a impressão de que o computador possui uma personalidade.

Chatterbots vêm sendo desenvolvidos para diversos propósitos, como a concepção de tutores virtuais em sistemas de ensino assistido a distância, assistentes virtuais pra atividades genéricas, teste de Turing, entre outras. Em todos os cenários citados espera-se que o chatterbot se comporte de forma natural, fazendo com que sua interação com o usuário seja simples e agradável. Os chatterbots aproximam os usuários da aplicação, por meio de uma interação muito aproximada à da linguagem natural, tentando fazê-lo acreditar que ele interage com um ser humano (propósito do Teste de Turing).

Para relatar esta pesquisa que está em andamento, além desta seção de Introdução, o artigo está estruturado da seguinte forma: a Seção 2 detalha a linguagem 
AIML, a Seção 3 detalha a linguagem EmotionML, a Seção 4 descreve a proposta e apresenta a arquitetura deste trabalho. A Seção 5 apresenta os resultados parciais, as conclusões e o prosseguimento desta pesquisa.

\section{AIML}

Uma das linguagens mais comuns para o desenvolvimento de chatterbots é a AIML [AIML 2014], que foi desenvolvida por Richard Wallace e pela comunidade do agora conhecido como A.L.I.C.E. AI Foundation (2001). É de fácil entendimento e amplamente utilizada para fazer quaisquer agentes inteligentes se comunicarem com seus usuários de maneira natural, baseada em XML com um conjunto de tags simples e intuitivo. Seu funcionamento básico é como em um interpretador recebendo estímulos / interações que estão registrados e caracterizados por meio de tags e possuem formas pré-definidas de reação a cada interação esperada.

O AIML apesar de permitir construir um chatterbot rico em reações e características é limitado ao se considerar alguns problemas como a falta de variação de reações durante a conversação visto que uma mesma resposta vai estar sempre relacionada a um padrão de entrada e também como citada abaixo. Sobre esta particularidade, Mikic e Burguillo [Mikic e Burguillo 2012] declararam:

"Um dos maiores problemas que o chatterbot mostra é a aleatoriedade quando escolhe entre muitas possibilidades de resposta para uma única questão, sem considerar a conversa em nenhum contexto. Esta situação resulta algumas vezes em respostas inconsistentes...".

Os seres humanos em sua conversação possuem essas diversidades de reações visto que a todo o momento inferem muitas informações de aspectos espaciais e emocionais, mas principalmente esta última visto que as características emocionais muitas vezes ditam muito das interações interpessoais.

\section{EmotionML}

Segundo Schröder e Baggia (2011) os sistemas computadorizados, à medida que eles podem reconhecer ou simular informações relacionadas a emoções, precisam de um formato de representação. Ainda segundo os autores se muitos componentes trabalham colaborativamente, o formato da informação deve ser bem definido.

Com esse propósito foi criada uma a linguagem EmotionML para representar emoções [EmotionML 2014]. EmotionML foi concebida considerando três modos de 
utilização: anotação manual de emoções observadas, detecção de emoções por meio do treinamento de classificadores para reconhecer automaticamente emoções e geração de emoções por meio de uma aplicação que faça a relação entre os dados emocionais e as reações ao meio. Assim consideramos a utilização da EmotionML uma segunda linguagem de marcação em XML que tem como finalidade tratamento e definição de características emocionais.

Como proposto por Charfuelan e Steiner (2013) o controle da expressividade em termos de diferentes emoções possíveis pode ser feito para vozes que foram treinadas com dados de diferentes estilos emocionais, onde variações de saídas sobre TTS (text-to-speech) são formuladas utilizando EmotionML, criando aspectos emocionais para serem aplicados na criação de audiobooks.

\section{Proposta}

A partir desse problema nos chatterbots propomos dar características mais humanas em sua conversação, criando novos aspectos e parâmetros a serem considerados durante a conversação. Neste artigo propomos um interpretador que faça uso das regras descritas pelo AIML unidas com os atributos de caracterização das emoções do EmotionML compondo uma linguagem mista AIML e EmotionML, provisoriamente denominada MindML que provenha dados emocionais resultantes das interações da conversação.

Um exemplo da combinação de AIML e EmotionML é mostrado na Figura 1. aqui $</$ li $>$

<emo:category name="interest" value="0.8"/> <li >Oi! É um prazer ter você

$<$ emo:category name="anger" value="0.8"/> <li $>$ Que é!? $</$ li $>$

$<$ emo:category name="worried" value="0.4"/> <li $>$ Oi! Precisando de aiuda? $</ \mathrm{li}>$

Figura 1. Exemplo de união de tag caracteristica do EmotionML num formato AIML

Além da definição formal de como o chatterbot será escrito é necessário considerar características da interpretação e tratamento das emoções durante sua operação conforme é mostrado na Figura 2. Nesse sentido, consideraremos algumas características que são os "traços de personalidade" e os aspectos sentidos no contexto, que afetam os nós emocionais gerando o "comportamento" do chatterbot. 


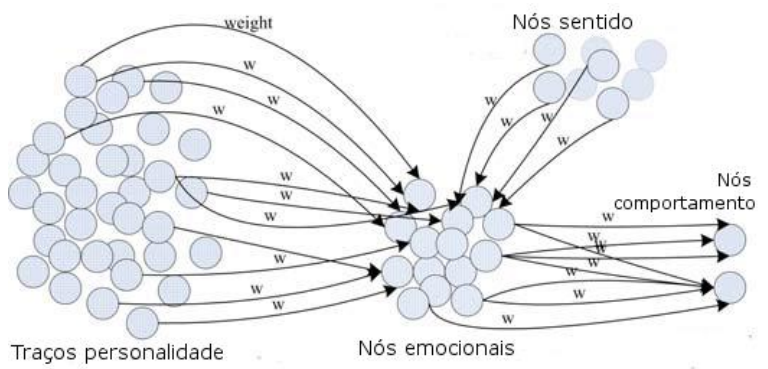

Figura 2. Fatores que agem sobre as emoções (Adaptado de Eladhari 2014)

Para Eladhari (2014), o comportamento pode ser visto como " ponta do iceberg" de emoções subjacentes. Assim como definido os nós de comportamento representam os aspectos internos e externos, que dividem a forma como as emoções podem ser percebidas. As emoções que atuam sobre estes comportamentos estão definidas de acordo com a classificação proposta pelo autor, conforme apresentado na Figura 3:



Figura 3. Emoções e suas implicações e pesos nos aspectos comportamentais (Adaptado de Eladhari 2014)

O sistema funcionará sob uma máquina Java que processará os arquivos de configuração do Chatterbot e receberá por meio de texto a interação do usuário, que processará os dados da conversação e suas implicações emocionais e responderá de forma coerente por meio de texto e expressões físicas por meio de um avatar animado.

A arquitetura do chatterbot é apresentada na Figura 4.

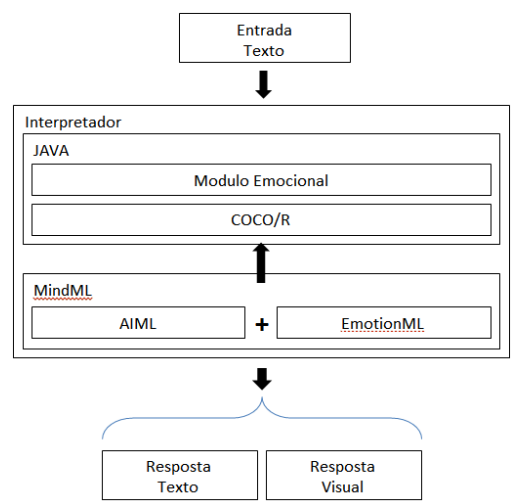

Figura 4. Arquitetura de Funcionamento do Chatterbot 
A arquitetura será organizada, e incluirá uma área onde serão exibidas questões relativas à disciplina em que o chatterbot estiver especializado. $\mathrm{O}$ conjunto comporá uma interface agradável e de fácil entendimento que atuara auxiliando o usuário possibilitando uma interação facilitada que ajudará no aprendizado e resposta das questões.

\section{Conclusões Parciais}

Concluímos após o estudo que o aumento das possibilidades é visivelmente um fator promissor para a adição de personalidade aos chatterbots fazendo-os ter reações situacionais, dotando-os de habilidades de considerar o contexto em que se encaixam.

Após o desenvolvimento de alguns protótipos da versão do EmotionML modificada, estamos desenvolvendo o interpretador que fará toda a avaliação e controle emocional do chatterbot, melhorando as interações já que considerando o contexto gera novas capacidades de tratamentos com os usuários fazendo-os sentirem-se mais confortáveis com o sistema e interessados em usá-lo.

\section{Referências}

A.L.I.C.E. AI Foundation. Artificial Intelligence Markup Language (AIML). Disponível em: http://www.alicebot.org/TR/2005/WD-aiml/.

Charfuelan, M., Steiner, I. (2013). Expressive speech synthesis in MARY TTS using audiobook data and EmotionML. Interspeech, ISCA, p. 1564-1568.

Eladhari, M. P. (2014). The Mind Module-Using an Affectand Personality Computational Modelas a Game-Play Element. IEEE Transactions on Affective Computing, 5(1), p. 3-16.

Mikic, F., Burguillo, J. (2012). Using tags in an AIML-based chatterbot to improve its knowledge. Computer Science, North America, 13, sep. 2012. Disponível em: http://journals.agh.edu.pl/csci/article/view/20.

Nichele, M., Jaques, P. A. (2013). Criação de um tradutor XML para a linguagem de marcações sobre emoção EmotionML. Revista Brasileira de Computação Aplicada, $5(2), 55-68$.

Schröder, M., \& Baggia, P. et al (2011). EmotionML-an upcoming standard for representing emotions and related states. 4th international conference on Affective computing and intelligent interaction - Volume Part I (ACII'11), Vol. Part I. Springer-Verlag, Berlin, Heidelberg, 316-325

Sganderla, R. B., Ferrari, D. N., \& Geyer, C. F. R. (2003). BonoBOT: Um Chatterbot para Interação com Usuários em um Sistema Tutor Inteligente. XIV Simpósio Brasileiro de Informática na Educação, 2-10.

W3C. Emotion Markup Language (EmotionML) 1.0. Disponível em http://www.w3.org/TR/emotionml/. 